\title{
TWO STEP ALGORITHM FOR SOLVING REGULARIZED GENERALIZED MIXED VARIATIONAL INEQUALITY PROBLEM
}

\author{
Kaleem Raza Kazmi, Faizan Ahmad Khan, and Mohammad Shahza
}

\begin{abstract}
In this paper, we consider a new class of regularized (nonconvex) generalized mixed variational inequality problems in real Hilbert space. We give the concepts of partially relaxed strongly mixed monotone and partially relaxed strongly $\theta$-pseudomonotone mappings, which are extension of the concepts given by Xia and Ding [19], Noor [13] and Kazmi et al. [9]. Further we use the auxiliary principle technique to suggest a two-step iterative algorithm for solving regularized (nonconvex) generalized mixed variational inequality problem. We prove that the convergence of the iterative algorithm requires only the continuity, partially relaxed strongly mixed monotonicity and partially relaxed strongly $\theta$-pseudomonotonicity. The theorems presented in this paper represent improvement and generalization of the previously known results for solving equilibrium problems and variational inequality problems involving the nonconvex (convex) sets, see for example Noor [13], Pang et al. [14], and Xia and Ding [19].
\end{abstract}

\section{Introduction}

Variational inequality theory, introduced by Stampacchia [18], has become a rich source of inspiration and motivation for the study of a large number of problems arising in economics, finance, transportation, network and structural analysis, elasticity and optimization. The ideas and techniques of this theory are being used in a variety of diverse areas and proved to be productive and innovative, see $[2,6-8,12,15]$. It is worth mentioning that most of the results regarding the existence and iterative approximation of solutions to variational

Received January 23, 2009; Revised September 14, 2009.

2000 Mathematics Subject Classification. 49J30, 90C30.

Key words and phrases. regularized generalized mixed variational inequality problem, auxiliary problem, two-step iterative algorithm, convergence analysis, partially relaxed strongly mixed monotone mapping, regularized partially relaxed strongly $\theta$-pseudomonotone mapping, skew-symmetric function.

The second author was supported by NBHM, Department of Atomic Energy, Government of India under Grants-in-aid for Post-doctoral fellowship (Reference no. 40/2/2007-R\&D II/3910). 
inequality problems have been investigated and considered so far refer to the case where the underlying set is a convex set.

In recent years, the concept of convex set has been generalized in many directions, which has potential and important applications in various fields. A significant generalization of convex set is the introduction of uniformly proxregular (smooth) set, see $[5,16,17]$. It is known that uniformly prox-regular sets are nonconvex sets and include convex sets as special case.

Very recently, Bounkhel et al. [4], Noor [13], Moudafi [10], and Pang et al. [14] have considered the variational inequality problems and equilibrium problems over these nonconvex sets. They suggested and analyzed some projection type iterative algorithms by using the prox-regular technique and auxiliary principle technique.

Motivated by the recent research going on in this area, we introduce a variational inequality problem, which shall be called regularized (nonconvex) generalized mixed variational inequality problem (in short, RGMVIP) in real Hilbert space. We give the concepts of partially relaxed strongly mixed monotone and regularized partially relaxed strongly $\theta$-pseudomonotone mappings, which are extension of the concepts given by Xia and Ding [19], Noor [13] and Kazmi et al. [9]. Further, we use the auxiliary principle technique to suggest a twostep iterative algorithm for approximating the solution of RGMVIP. We prove that the convergence of the iterative algorithm requires only the continuity, partially relaxed strongly mixed monotonicity and partially relaxed strongly $\theta$ pseudomonotonicity. The theorems presented in this paper represent improvement and generalization of the previously known results for solving equilibrium problems and variational inequality problems involving the nonconvex (convex) sets, see for example Noor [13], Pang et al. [14], and Xia and Ding [19].

\section{Preliminaries}

Let $H$ be a real Hilbert space whose norm and inner product are denoted by $\|\cdot\|$ and $\langle\cdot, \cdot\rangle$, respectively. Let $K$ be a nonempty closed set in $H$, not necessarily convex. Let $C B(H)$ be a family of all nonempty, closed and bounded subsets of $H$, and let $M(\cdot, \cdot)$ be the Hausdorff metric on $C B(H)$ defined by

$$
M(C, D)=\max \left\{\sup _{x \in C} \inf _{y \in D} d(x, y), \sup _{y \in D} \inf _{x \in C} d(x, y)\right\}, C, D \in C B(H) .
$$

First, we recall the following well-known concepts from nonlinear convex analysis, see $[5,16,17]$.

Definition 2.1. The proximal normal cone of $K$ at $u \in H$ is given by

$$
N^{P}(K ; u):=\left\{\xi \in H: u \in P_{K}[u+\alpha \xi]\right\},
$$

where $\alpha>0$ is a constant and

$$
P_{K}[u]=\left\{u^{*} \in K: d_{K}(u)=\left\|u-u^{*}\right\|\right\},
$$


where $d_{K}(u)$ is the usual distance function to the subset $K$, that is,

$$
d_{K}(u)=\inf _{v \in K}\|v-u\| \text {. }
$$

The proximal normal cone $N^{P}(K ; u)$ has the following characterization.

Lemma 2.1. Let $K$ be a nonempty closed subset of $H$. Then $\xi \in N^{P}(K ; u)$ if and only if there exists a constant $\alpha>0$ such that

$$
\langle\zeta, v-u\rangle \leq \alpha\|v-u\|^{2}, \forall v \in K
$$

Definition 2.2. The Clarke normal cone, denoted by $N^{C}(K ; u)$, is defined as

$$
N^{C}(K ; u)=\overline{\operatorname{co}}\left[N^{P}(K ; u)\right],
$$

where $\overline{\mathrm{co}} A$ means the closure of the convex hull of $A$.

Poliquin et al. [16] and Clarke et al. [5] have introduced and studied a new class of nonconvex sets, which are called uniformly prox-regular sets. This class of uniformly prox-regular sets has played an important role in many nonconvex applications such as optimization, dynamic systems and differential inclusions. In particular, we have:

Definition 2.3. For a given $r \in(0, \infty]$, a subset $K$ is said to be normalized uniformly $r$-prox-regular if and only if every nonzero proximal normal to $K$ can be realized by any $r$-ball, that is, $\forall u \in K$ and $0 \neq \xi \in N_{P}(K ; u)$ with $\|\xi\|=1$, one has

$$
\langle\xi, v-u\rangle \leq \frac{1}{2 r}\|v-u\|^{2}, \forall v \in K
$$

It is clear that the class of normalized uniformly prox-regular sets is sufficiently large to include the class of convex sets, $p$-convex sets, $C^{1,1}$ submanifolds (possibly with boundary) of $H$, the images under a $C^{1,1}$ diffeomorphism of convex sets and many other nonconvex sets, see $[5,16,17]$. It is clear that if $r=\infty$, then uniformly $r$-prox-regularity of $K$ is equivalent to the convexity of $K$.

It is known that if $K$ is a uniformly prox-regular set, the proximal normal cone $N_{P}(K ; u)$ is closed as a set-valued mapping. Thus, we have $N_{C}(K ; u)=$ $N_{P}(K ; u)$.

From now onward, the set $K$ is uniformly $r$-prox-regular set, unless otherwise specified. For given a nonlinear differentiable bifunction $b: H \times H \rightarrow \mathbb{R} \cup\{+\infty\}$, a nonlinear bifunction $F: H \times H \times H \rightarrow \mathbb{R}$; a nonlinear mapping $N: H \times$ $H \rightarrow H$ and three set-valued mappings $T, A, B: H \rightarrow C B(H)$, we consider the following regularized generalized mixed variational inequality problem (in short, RGMVIP):

Find $u \in K, x \in T(u), y \in A(u), z \in B(u)$ such that

(2.1) $F(x, v, u)+\langle N(y, z), v-u\rangle+b(v, u)-b(u, u)+\frac{1}{2 r}\|v-u\|^{2} \geq 0, \forall v \in K$.

\section{Some special cases of RGMVIP (2.1):}


I. If $\gamma=\frac{1}{2 r}=0$, then uniformly prox-regular set $K$ becomes the convex set $K$ and consequently RGMVIP (2.1) reduces to the problem of finding $u \in K, x \in T(u), y \in A(u), z \in B(u)$ such that

$$
F(x, v, u)+\langle N(y, z), v-u\rangle+b(v, u)-b(u, u) \geq 0, \forall v \in K,
$$

which appears to be new one.

II. In (I), if $N(y, z) \equiv \mathbf{0} \forall y, z \in H$, RGMVIP (2.1) reduces to the problem of finding $u \in K, x \in T(u)$ such that

$$
F(x, v, u)+b(v, u)-b(u, u) \geq 0, \forall v \in K,
$$

which is known as mixed quasi-equilibrium problem and has been studied by Noor [12] and Xia and Ding [19].

III. If $F(x, v, u) \equiv \mathbf{0} \forall u, v, x \in H$, RGMVIP (2.1) reduces to the problem of finding $u \in K, y \in A(u), z \in B(u)$ such that

$$
\langle N(y, z), v-u\rangle+b(v, u)-b(u, u)+\frac{1}{2 r}\|v-u\|^{2} \geq 0, \forall v \in K,
$$

which appears to be new one.

IV. If $N(y, z) \equiv \mathbf{0} \forall y, z \in H ; T: H \rightarrow H$ is a single valued mapping and $\frac{1}{2 r}=0$, RGMVIP (2.1) reduces to the problem of finding $u \in K$ such that

$$
F(T u, v, u)+b(v, u)-b(u, u)+\frac{1}{2 r}\|v-u\|^{2} \geq 0, \forall v \in K
$$

which is known as regularized mixed quasi-equilibrium problem and has been studied by Noor [13].

Now, we give the following concepts and known results which are used in the sequel.

Definition 2.4. Let $T, A, B: H \rightarrow C B(H) ; F: H \times H \times H \rightarrow \mathbb{R}$ and let $b: H \times H \rightarrow \mathbb{R} \cup\{+\infty\}$. Then, for all $u, v, w \in K, x_{1} \in T(u), x_{2} \in T(v), y_{1} \in$ $A(u), y_{2} \in A(u), z_{1} \in B(u), z_{2} \in B(v)$,

(i)[9] $N$ is said to be mixed monotone with respect to $A$ and $B$ if

$$
\left\langle N\left(y_{1}, z_{1}\right)-N\left(y_{2}, z_{2}\right), u-v\right\rangle \geq 0 ;
$$

(ii)[9] $N$ is said to be $\mu$-partially relaxed strongly mixed monotone with respect to $A$ and $B$ if there exists a constant $\eta>0$ such that

$$
\left\langle N\left(y_{1}, z_{1}\right)-N\left(y_{2}, z_{2}\right), w-v\right\rangle \geq-\mu\|w-u\|^{2}
$$

(iii)[19] $T$ is said to be $M$-continuous if $\left\{u_{n}\right\} \subset H$ and $u_{n} \rightarrow u$, then $T\left(u_{n}\right) \rightarrow$ $T(u)$ under the Hausdorff metric $M$ on $C B(H)$;

(iv)[19] $F$ is said to be monotone with respect to $T$ if

$$
F\left(x_{1}, v, u\right)+F\left(x_{2}, u, v\right) \leq 0 ;
$$

(v)[19] $F$ is said to be pseudomonotone with respect to $T$ if

$$
F\left(x_{2}, v, u\right) \geq 0 \text { implies } F\left(x_{1}, u, v\right) \leq 0 ;
$$


(vi)[19] $F$ is said to be $\delta$-partially relaxed strongly monotone with respect to $T$ if there exists a constant $\delta>0$ such that

$$
F\left(x_{1}, v, u\right)+F\left(x_{2}, w, v\right) \leq \delta\|w-u\|^{2} ;
$$

(vii)[19] $F$ is said to be $\sigma$-partially relaxed strongly pseudomonotone with respect to $T$ if there exists a constant $\sigma>0$ such that

$$
F\left(x_{2}, w, v\right) \geq 0 \text { implies } F\left(x_{1}, v, u\right) \leq \sigma\|w-u\|^{2} ;
$$

(viii) $\quad F$ is said to be regularized $\gamma$-partially relaxed strongly $\theta$-pseudomonotone with respect to $T$, where $\theta$ is a real-valued multivariable function, if there exists a constant $\gamma>0$ such that

$$
F\left(x_{2}, w, v\right)+\theta+\frac{1}{2 r}\|v-u\|^{2} \geq 0
$$

implies

$$
F\left(x_{1}, v, u\right)-\theta+\frac{1}{2 r}\|v-u\|^{2} \leq \gamma\|w-u\|^{2}
$$

(ix)[19] $b$ is said to be skew-symmetric if

$$
b(u, u)-b(u, v)-b(v, u)+b(v, v) \geq 0, \forall u, v \in H .
$$

Remark 2.1. (1) If $w=u$, then partially relaxed strongly mixed monotonicity of $N$ reduces to the mixed monotonicity of $N$.

(2) Regularized $\gamma$-partially relaxed strongly $\theta$-psedomonotonicity of $F$ generalize the concepts of partially relaxed strongly jointly pseudomonotonicity of $F$ given by Xia and Ding [19] and $\theta$-pseudomonotonicity of $F$ given by Kazmi et al. [9].

(3) We note that if skew-symmetric bifunction $b$ is bilinear, then $b(u, u) \geq$ $0, \forall u \in H$.

Lemma 2.2. For all $u, v \in H$, we have

$$
2\langle u, v\rangle=\|u+v\|^{2}-\|u\|^{2}-\|v\|^{2} .
$$

\section{Main results}

In this section, we suggest and analyze a new iterative method for solving RGMVIP (2.1) by using the auxiliary principle technique.

For a given $u \in K, x \in T(u), y \in A(u), z \in B(u)$, consider the following auxiliary inequality problem (AVIP): Find $w \in K$ such that

$$
\begin{aligned}
\rho F(x, v, w) & +\langle\rho N(y, z)+w-u, v-w\rangle \\
& +\rho b(v, w)-\rho b(w, w)+\frac{\rho}{2 r}\|v-w\|^{2} \geq 0, \forall v \in K,
\end{aligned}
$$

where $r \in(0, \infty]$ and $\rho>0$ is a constant.

We note that if $w=u$, then clearly $w$ is a solution of RGMVIP (2.1). This observation and Nadler's technique [11] enable us to suggest and analyze the 
following two-step iterative algorithm for finding the approximate solution of RGMVIP (2.1).

Iterative Algorithm 3.1. For given $u_{0} \in K, x_{0} \in T\left(u_{0}\right), y_{0} \in A\left(u_{0}\right), z_{0} \in$ $B\left(u_{0}\right)$, compute the approximate solution $\left(u_{n}, w_{n}, x_{n}, y_{n}, z_{n}\right)$ by the iterative schemes:

$$
\begin{gathered}
\rho F\left(\xi_{n}, v, w_{n}\right)+\left\langle\rho N\left(\eta_{n}, \gamma_{n}\right)+u_{n+1}-w_{n}, v-u_{n+1}\right\rangle+\rho b\left(v, u_{n+1}\right) \\
-\rho b\left(u_{n+1}, u_{n+1}\right)+\frac{\rho}{2 r}\left\|v-w_{n}\right\|^{2} \geq 0, \forall v \in K \\
\xi_{n} \in T\left(w_{n}\right):\left\|\xi_{n+1}-\xi_{n}\right\| \leq\left(1+(1+n)^{-1}\right) M\left(T\left(w_{n+1}\right), T\left(w_{n}\right)\right) ; \\
\eta_{n} \in A\left(w_{n}\right):\left\|\eta_{n+1}-\eta_{n}\right\| \leq\left(1+(1+n)^{-1}\right) M\left(A\left(w_{n+1}\right), A\left(w_{n}\right)\right) ; \\
\gamma_{n} \in B\left(w_{n}\right):\left\|\gamma_{n+1}-\gamma_{n}\right\| \leq\left(1+(1+n)^{-1}\right) M\left(B\left(w_{n+1}\right), B\left(w_{n}\right)\right) ; \\
\beta F\left(x_{n}, v, u_{n}\right)+\left\langle\beta N\left(y_{n}, z_{n}\right)+w_{n}-u_{n}, v-w_{n}\right\rangle \\
+\beta b\left(v, w_{n}\right)-\beta b\left(w_{n}, w_{n}\right)+\frac{\beta}{2 r}\left\|v-u_{n}\right\|^{2} \geq 0, \forall v \in K \\
x_{n} \in T\left(u_{n}\right):\left\|x_{n+1}-x_{n}\right\| \leq\left(1+(1+n)^{-1}\right) M\left(T\left(u_{n+1}\right), T\left(u_{n}\right)\right) ; \\
y_{n} \in A\left(u_{n}\right):\left\|y_{n+1}-y_{n}\right\| \leq\left(1+(1+n)^{-1}\right) M\left(A\left(u_{n+1}\right), A\left(u_{n}\right)\right) ; \\
z_{n} \in B\left(u_{n}\right):\left\|z_{n+1}-z_{n}\right\| \leq\left(1+(1+n)^{-1}\right) M\left(B\left(u_{n+1}\right), B\left(u_{n}\right)\right),
\end{gathered}
$$

where $n=1,2, \ldots$ and $\rho, \beta>0$ are constants.

For convergence analysis of Iterative Algorithm 3.1, we have the following theorem.

Theorem 3.1. Let $(u, w, x, y, z)$, where $u, w \in K, x \in T(u), y \in A(u), z \in$ $B(u)$ be a solution of $R G M V I P(2.1)$ and let $\left(u_{n}, w_{n}, x_{n}, y_{n}, z_{n}\right)$ be an approximate solution obtained by Iterative Algorithm 3.1. Let $F$ be regularized $\alpha$-partially relaxed strongly $\theta$-pseudomonotone with respect to $T$, where $\theta$ is a real-valued multivariable function defined as

$$
\theta(y, z, u, v)=\langle N(y, z), v-u)\rangle+b(v, u)+b(u, u), \forall y, z, u, v \in H
$$

let $N$ be $\mu$-partially relaxed strongly mixed monotone with respect to $A$ and $B$, and let $b$ be skew symmetric. Then

(3.6) $\left\|u_{n+1}-u\right\|^{2} \leq\left\|u_{n}-u\right\|^{2}-(1-2 \rho(\alpha+\mu))\left(1+\frac{\rho}{r}\right)^{-1}\left\|u_{n+1}-w_{n}\right\|^{2}$,

(3.7) $\left\|w_{n}-u\right\|^{2} \leq\left\|w_{n-1}-u\right\|^{2}-(1-2 \beta(\alpha+\mu))\left(1+\frac{\beta}{r}\right)^{-1}\left\|w_{n}-u_{n}\right\|^{2}$, where $0<\rho, \beta<\frac{1}{2(\alpha+\mu)}$ and $r \in(0, \infty]$. 
Proof. By hypothesis, $(u, v, x, y, z)$ satisfies

$\rho F(x, v, u)+\langle\rho N(y, z), v-u\rangle+\rho b(v, u)-\rho b(u, u)+\frac{\rho}{2 r}\|v-u\|^{2} \geq 0, \forall v \in K$,

$\beta F(x, v, u)+\langle\beta N(y, z), v-u\rangle+\beta b(v, u)-\beta b(u, u)+\frac{\beta}{2 r}\|v-u\|^{2} \geq 0, \forall v \in K$, where $r \in(0, \infty]$ and $\rho, \beta>0$ are constants.

Now, taking $v=u_{n+1}$ in (3.8), we have

$$
\begin{aligned}
\rho F\left(x, u_{n+1}, u\right) & +\left\langle\rho N(y, z), u_{n+1}-u\right\rangle \\
& +\rho b\left(u_{n+1}, u\right)-\rho b(u, u)+\frac{\rho}{2 r}\left\|u_{n+1}-u\right\|^{2} \geq 0 .
\end{aligned}
$$

Since $F$ is regularized $\alpha$-partially relaxed strongly $\theta$-pseudomonotone with respect to $T$, then (3.10) implies that

$$
\begin{aligned}
\rho F\left(\xi_{n}, u, w_{n}\right) & +\left\langle\rho N(y, z), u-u_{n+1}\right\rangle+\rho b(u, u) \\
& -\rho b\left(u_{n+1}, u\right)+\frac{\rho}{2 r}\left\|u_{n+1}-u\right\|^{2} \leq \rho \alpha\left\|u_{n+1}-w_{n}\right\|^{2} .
\end{aligned}
$$

Taking $v=u$ in (3.2), we obtain

$$
\begin{aligned}
\rho F\left(\xi_{n}, u, w_{n}\right) & +\left\langle\rho N\left(\eta_{n}, \gamma_{n}\right)+u_{n+1}-w_{n}, u-u_{n+1}\right\rangle+\rho b\left(u, u_{n+1}\right) \\
& -\rho b\left(u_{n+1}, u_{n+1}\right)+\frac{\rho}{2 r}\left\|u-w_{n}\right\|^{2} \geq 0 .
\end{aligned}
$$

From (3.11) and (3.12), we have

$$
\begin{aligned}
& \left\langle u_{n+1}-w_{n}, u-u_{n+1}\right\rangle \\
\geq & \rho\left\langle N\left(\eta_{n}, \gamma_{n}\right)-N(y, z), u_{n+1}-u\right\rangle \\
& +\frac{\rho}{2 r}\left\|u_{n+1}-u\right\|^{2}-\frac{\rho}{2 r}\left\|u-w_{n}\right\|^{2} \\
& +\rho\left[b(u, u)-b\left(u_{n+1}, u\right)-b\left(u, u_{n+1}\right)+b\left(u_{n+1}, u_{n+1}\right)\right] \\
& -\rho \alpha\left\|u_{n+1}-w_{n}\right\|^{2} .
\end{aligned}
$$

Since $N$ is $\mu$-partially relaxed strongly mixed monotone with respect to $A$ and $B$ and $b$ is skew symmetric, then (3.13) reduces to

$$
\begin{aligned}
& \left\langle u_{n+1}-w_{n}, u-u_{n+1}\right\rangle \\
\geq & -\rho \mu\left\|u_{n+1}-w_{n}\right\|^{2}+\frac{\rho}{2 r}\left\|u_{n+1}-u\right\|^{2}-\frac{\rho}{2 r}\left\|u-w_{n}\right\|^{2}-\rho \alpha\left\|u_{n+1}-w_{n}\right\|^{2} \\
\geq & -\rho(\alpha+\mu)\left\|u_{n+1}-w_{n}\right\|^{2}+\frac{\rho}{2 r}\left\|u_{n+1}-u\right\|^{2}-\frac{\rho}{2 r}\left\|u-w_{n}\right\|^{2} .
\end{aligned}
$$

By Lemma 2.2, we have

(3.15) $\left\langle u_{n+1}-w_{n}, u-u_{n+1}\right\rangle=\frac{1}{2}\left[\left\|u-w_{n}\right\|^{2}-\left\|u_{n+1}-w_{n}\right\|^{2}-\left\|u-u_{n+1}\right\|^{2}\right]$. 
Combining (3.14) and (3.15), we have

$\left(1+\frac{\rho}{r}\right)\left\|u_{n+1}-u\right\|^{2} \leq\left(1+\frac{\rho}{r}\right)\left\|u-w_{n}\right\|^{2}-(1-2 \rho(\alpha+\mu))\left\|u_{n+1}-w_{n}\right\|^{2}$,

which implies that

(3.16) $\left\|u_{n+1}-u\right\|^{2} \leq\left\|u-w_{n}\right\|^{2}-(1-2 \rho(\alpha+\mu))\left(1+\frac{\rho}{r}\right)^{-1}\left\|u_{n+1}-w_{n}\right\|^{2}$.

Now, taking $v=w_{n}$ in (3.9), we have

$\beta F\left(x, w_{n}, u\right)+\left\langle\beta N(y, z), w_{n}-u\right\rangle+\beta b\left(w_{n}, u\right)-\beta b(u, u)+\frac{\beta}{2 r}\left\|w_{n}-u\right\|^{2} \geq 0$.

Since $F$ is regularized $\alpha$-partially relaxed strongly $\theta$-pseudomonotone with respect to $T$, then (3.17) implies that

$$
\begin{aligned}
\beta F\left(x_{n}, u, u_{n}\right) & +\left\langle\beta N(y, z), u-w_{n}\right\rangle+\beta b(u, u) \\
& -\beta b\left(w_{n}, u\right)+\frac{\beta}{2 r}\left\|w_{n}-u\right\|^{2} \leq \beta \alpha\left\|w_{n}-u_{n}\right\|^{2} .
\end{aligned}
$$

Taking $v=u$ in (3.4), we obtain

$$
\begin{aligned}
\beta F\left(x_{n}, u, u_{n}\right) & +\left\langle\beta N\left(y_{n}, z_{n}\right)+w_{n}-u_{n}, u-w_{n}\right\rangle \\
& +\beta b\left(u, w_{n}\right)-\beta b\left(w_{n}, w_{n}\right)+\frac{\beta}{2 r}\left\|u-u_{n}\right\|^{2} \geq 0 .
\end{aligned}
$$

Since $N$ is $\mu$-partially relaxed strongly mixed monotone with respect to $A$ and $B$ and $b$ is skew symmetric, then it follows from (3.18) and (3.19) that

(3.20) $\left\langle w_{n}-u_{n}, u-w_{n}\right\rangle \geq-\beta(\alpha+\mu)\left\|w_{n}-u_{n}\right\|^{2}+\frac{\beta}{2 r}\left\|u-w_{n}\right\|^{2}-\frac{\beta}{2 r}\left\|u-u_{n}\right\|^{2}$.

Again, by Lemma 2.2, we have

$$
\left\langle w_{n}-u_{n}, u-w_{n}\right\rangle=\frac{1}{2}\left[\left\|u_{n}-u\right\|^{2}-\left\|w_{n}-u_{n}\right\|^{2}-\left\|u-w_{n}\right\|^{2}\right] .
$$

Combining (3.20) and (3.21), we have

$$
\left(1+\frac{\beta}{r}\right)\left\|w_{n}-u\right\|^{2} \leq\left(1+\frac{\beta}{r}\right)\left\|u-u_{n}\right\|^{2}-(1-2 \beta(\alpha+\mu))\left\|w_{n}-u_{n}\right\|^{2},
$$

which implies that

$$
\left\|w_{n}-u\right\|^{2} \leq\left\|u-u_{n}\right\|^{2}-(1-2 \beta(\alpha+\mu))\left(1+\frac{\beta}{r}\right)^{-1}\left\|w_{n}-u_{n}\right\|^{2} .
$$

Since $0<\rho, \beta<\frac{1}{2(\alpha+\mu)}$, inequalities (3.16) and (3.22) imply that

$$
\left\|u_{n+1}-u\right\|^{2} \leq\left\|u-w_{n}\right\|^{2}
$$

and

$$
\left\|w_{n}-u\right\|^{2} \leq\left\|u-u_{n}\right\|^{2}
$$

and hence conclusion (3.6) and (3.7) hold. This completes the proof. 
Theorem 3.2. Let $H$ be a finite dimensional space. Let the mappings $b, F$, $N, T, A, B$ be the same as in Theorem 3.1 and let $F, N$ be continuous and $T, A, B$ be $M$-continuous mappings. If $0<\rho, \beta<\frac{1}{2(\alpha+\mu)}$, then the sequences $\left\{u_{n}\right\},\left\{w_{n}\right\},\left\{x_{n}\right\},\left\{y_{n}\right\},\left\{z_{n}\right\}$ generated by Iterative Algorithm 3.1 converge strongly to a solution of RGMVIP (2.1).

Proof. Let $u \in K, x \in T(u), y \in A(u), z \in B(u)$ be a solution of RGMVIP (2.1), inequalities (3.6) and (3.7) hold. From (3.6) and (3.7), it follows that the sequences $\left\{\left\|u_{n}-u\right\|\right\}$ and $\left\{\left\|w_{n}-u\right\|\right\}$ are non-increasing, and consequently $\left\{u_{n}\right\}$ and $\left\{w_{n}\right\}$ are bounded. Furthermore, we have

$$
\begin{gathered}
\sum_{n=0}^{\infty}(1-2 \rho(\alpha+\mu))\left(1+\frac{\rho}{r}\right)^{-1}\left\|u_{n+1}-w_{n}\right\|^{2} \leq\left\|u-u_{0}\right\|^{2}, \\
\sum_{n=0}^{\infty}(1-2 \beta(\alpha+\mu))\left(1+\frac{\beta}{r}\right)^{-1}\left\|w_{n}-u_{n}\right\|^{2} \leq\left\|u-w_{0}\right\|^{2},
\end{gathered}
$$

which imply that $\lim _{n \rightarrow \infty}\left\|u_{n+1}-w_{n}\right\|^{2}=0$ and $\lim _{n \rightarrow \infty}\left\|w_{n}-u_{n}\right\|^{2}=0$.

Since

$$
\left\|u_{n+1}-u_{n}\right\| \leq\left\|u_{n+1}-w_{n}\right\|+\left\|w_{n}-u_{n}\right\|, \forall n \geq 0
$$

we have

$$
\lim _{n \rightarrow \infty}\left\|u_{n+1}-u_{n}\right\|=0 .
$$

Since $\left\{u_{n}\right\}$ is bounded in $K,\left\{u_{n}\right\}$ has a subsequence $\left\{u_{n_{i}}\right\}$ converging to a point $u^{*} \in K$. Since $T, A, B$ are $M$-continuous on $H$, it follows from Proposition 1.5.2 [1] that $T, A, B$ are upper semi-continuous on $H$. Since $x_{n} \in$ $T\left(u_{n}\right), y_{n} \in A\left(u_{n}\right), z_{n} \in B\left(u_{n}\right)$ for all $n \geq 0$, it follows from Proposition 11.11 [2] that there are subsequences $\left\{x_{n_{i_{j}}}\right\} \subset\left\{x_{n_{i}}\right\},\left\{y_{n_{i_{j}}}\right\} \subset\left\{y_{n_{i}}\right\},\left\{z_{n_{i_{j}}}\right\} \subset\left\{z_{n_{i}}\right\}$ such that $x_{n_{i_{j}}} \rightarrow x^{*} \in T\left(u^{*}\right), y_{n_{i_{j}}} \rightarrow y^{*} \in A\left(u^{*}\right), z_{n_{i_{j}}} \rightarrow z^{*} \in B\left(u^{*}\right)$. By (3.4), we have

$$
\begin{aligned}
\beta F\left(x_{n_{i_{j}}}, v, u_{n_{i_{j}}}\right) & +\left\langle\beta N\left(y_{n_{i_{j}}}, z_{n_{i_{j}}}\right)+w_{n_{i_{j}}}-u_{n_{i_{j}}}, v-w_{n_{i_{j}}}\right\rangle+\beta b\left(v, w_{n_{i_{j}}}\right) \\
& -\beta b\left(w_{n_{i_{j}}}, w_{n_{i_{j}}}\right)+\frac{\beta}{2 r}\left\|v-u_{n_{i_{j}}}\right\|^{2} \geq 0, \quad \forall v \in K .
\end{aligned}
$$

Continuity of $F, N, b$, and (3.23) implies that

$$
F\left(x^{*}, v, u^{*}\right)+\left\langle N\left(y^{*}, z^{*}\right), v-u^{*}\right\rangle+b\left(v, u^{*}\right)-b\left(u^{*}, u^{*}\right) \geq 0, \forall v \in K .
$$

Thus $u^{*} \in K, x^{*} \in T\left(u^{*}\right), y^{*} \in A\left(u^{*}\right), z^{*} \in B\left(u^{*}\right)$ is a solution of RGMVIP (2.1).

Now, we claim that the sequences $\left\{u_{n}\right\},\left\{x_{n}\right\},\left\{y_{n}\right\},\left\{z_{n}\right\}$ converges to $u^{*}, x^{*}$, $y^{*}, z^{*}$, respectively. In fact, since $\lim _{n \rightarrow \infty}\left\|u_{n+1}-u_{n}\right\|=0, \lim _{i \rightarrow \infty} u_{n_{i}}=u^{*}$ and

$\left\|u_{n}-u^{*}\right\| \leq\left\|u_{n}-u_{n+1}\right\|+\left\|u_{n+1}-u_{n+2}\right\|+\cdots+\left\|u_{n_{i-1}}-u_{n_{i}}\right\|+\left\|u_{n_{i}}-u^{*}\right\|$, 
we have $\lim _{n \rightarrow \infty} \quad u_{n}=u^{*}$. Since $T$ is $M$-continuous, by (3.5) we have $\lim _{n \rightarrow \infty}\left\|x_{n+1}-x_{n}\right\|=0$.

It follows from the inequality

$$
\left\|x_{n}-x^{*}\right\| \leq\left\|x_{n}-x_{n+1}\right\|+\left\|x_{n+1}-x_{n+2}\right\|+\cdots+\left\|x_{n_{i_{j}}}-x^{*}\right\|,
$$

that is, $x_{n} \rightarrow x^{*}$. Similarly, we have $y_{n} \rightarrow y^{*}$ and $z_{n} \rightarrow z^{*}$. This completes the proof.

\section{References}

[1] J. P. Aubin and A. Cellina, Differential Inclusions, Springer-Verlag, New York, 1984.

[2] E. Blum and W. Oettli, From optimization and variational inequalities to equilibrium problems, Math. Student 63 (1994), no. 1-4, 123-145.

[3] K. C. Border, Fixed Point Theorems with Applications to Economics and Game Theory, Cambridge University Press, Cambridge, 1985.

[4] M. Bounkhel, L. Tadj, and A. Hamdi, Iterative schemes to solve nonconvex variational problems, JIPAM. J. Inequal. Pure Appl. Math. 4 (2003), no. 1, Article 14, 14 pp.

[5] F. H. Clarke, Y. S. Ledyaev, R. J. Stern, and P. R. Wolenski, Nonsmooth Analysis and Control Theory, Graduate Texts in Mathematics, 178. Springer-Verlag, New York, 1998.

[6] F. Giannessi and A. Maugeri, Variational Inequalities and Network Equilibrium Problems, Plenum Press, New York, 1995.

[7] F. Giannessi, A. Maugeri, and P. M. Pardalos, Equilibrium Problems: Non-smooth Optimization and Variational Inequality Models, Kluwer Academic Publishers, Dordrecht, 2001.

[8] R. Glowinski, J. L. Lions, and R. Trémolières, Numerical Analysis of Variational Inequalities, North-Holland Publishing Co., Amsterdam-New York, 1981.

[9] K. R. Kazmi, A. Khaliq, and A. Raouf, Iterative approximation of solution of generalized mixed set-valued variational inequality problem, Math. Inequal. Appl. 10 (2007), no. 3, $677-691$.

[10] A. Moudafi, An algorithmic approach to prox-regular variational inequalities, Appl. Math. Comput. 155 (2004), no. 3, 845-852.

[11] S. B. Nadler, Jr., Multi-valued contraction mappings, Pacific J. Math. 30 (1969), 475488.

[12] M. A. Noor, Auxiliary principle technique for equilibrium problems, J. Optim. Theory Appl. 122 (2004), no. 2, 371-386.

[13] _ Regularized mixed quasi equilibrium problems, J. Appl. Math. Comput. 23 (2007), no. 1-2, 183-191.

[14] L.-P. Pang, J. Shen, and H.-S. Song, A modified predictor-corrector algorithm for solving nonconvex generalized variational inequality, Comput. Math. Appl. 54 (2007), no. 3, 319-325.

[15] M. Patriksson, Nonlinear Programming and Variational Inequality Problems, Kluwer Academic Publishers, Dordrecht, 1999.

[16] R. A. Poliquin and R. T. Rockafellar, Prox-regular functions in variational analysis, Trans. Amer. Math. Soc. 348 (1996), no. 5, 1805-1838.

[17] R. T. Rockafellor and R. J.-B. Wets, Variational Analysis, Springer-Verlag, Berlin, 1998.

[18] G. Stampacchia, Formes bilinéaires coercitives sur les ensembles convexes, C. R. Acad. Sci. Paris 258 (1964), 4413-4416.

[19] F. Q. Xia and X. P. Ding, Predictor-corrector algorithms for solving generalized mixed implicit quasi-equilibrium problems, Appl. Math. Comput. 188 (2007), no. 1, 173-179. 
KaLEem Raza KaZMi

Department of Mathematics

Aligarh Muslim University

Aligarh 202 002, IndiA

E-mail address: krkazmi@gmail.com

FAizan Ahmad Khan

Department of Mathematics

Aligarh Muslim University

Aligarh 202 002, IndiA

E-mail address: faizan_math@yahoo.com

MOHAmmad SHahza

Department of Applied Mathematics

Aligarh Muslim University

Aligarh 202 002, IndiA

E-mail address: shahzadamu@yahoo.co.in 